\title{
Brendan Gleeson
}

\section{Das $\gg$ Neuseeland-Wunder $\ll$ : Schicksal oder Märchen?}

\section{Eine wundersame Heilung}

In den letzten Jahren hat man den Europäern erzählt, daß in einem fernen und exotischen Land etwas Erstaunliches passiert sei - in Neuseeland, einem bislang eher unbekannten Vorposten des Britischen Empire. Alle möglichen Propheten, unter ihnen Ökonomen, Finanzjournalisten, Wirtschaftsführer und Politiker, sind weit gereist, um der Welt vom »Neuseeland-Wunder « zu berichten, der erstaunlichen Heilung einer schuldengeplagten und trägen Volkswirtschaft. Ihre Berichte wurden von den europäischen Medien wärmstens aufgenommen und auch von den nationalen Politikern, die gierig darauf waren, ein neues Gegengift für die sich verschärfenden Krankheiten des Spätkapitalismus zu entdecken.

Das »Neuseeland-Wunder" wird großspurig in Weltfinanz- und Regierungskreisen gepriesen - die OECD (1994) begrüßt die »Genesung «, die auf Jahre vernünftiger, aber schmerzlicher Reform folgen mußte, während Moody's Investors Services, die mächtige internationale Rating-Agentur, für »eine der ehrgeizigsten und umfassendsten Strukturreformen, die je in einem OECD-Land unternommen wurden«, schwärmt; es sei ein »günstiger Kreislauf« eines sich selbst regulierenden Wachstums in Gang gesetzt worden (zit. n. Kelsey 1995: 7). Das einflußreiche Weltwirtschaftsforum in Genf hat Neuseeland zum »neuen Star« der globalen Wettbewerbswirtschaft ausgerufen (The Australian Financial Review 30.5.96). Einige der überschwenglichsten Lobeshymnen kamen vom englischen Economist, der wiederholt zur $» g r u ̈ n d l i c h s t e n$ Wirtschaftsreform innerhalb der OECD« gratulierte, weil sie »ein Paradies für Marktwirtschaftler« geschaffen hätte, "wenn auch nicht für die Neuseeländer, die ihre Arbeit verloren haben « (zit. n. Kelsey 1995: 8). Wie Forer (1995) und Kelsey (1995) berichten, wurde die Begeisterung des Economist vom Wall Street Journal, von Torontos Globe and Mail, der britischen Times und der Financial Times geteilt. Auch viele der größeren australischen Zeitungen, 
besonders The Australian und The Australian Financial Review, sind unermüdliche Unterstützer des »Neuseeland-Wunders« gewesen.

Neuseelands Reformprogramm wird von seinen Befürwortern als 》Strukturanpassungsprogramm《 bezeichnet (im folgenden SAP). Nirgendwo in einem entwickelten Land wurden die ökonomischen Rezepte der ChicagoSchool energischer verfolgt als in Neuseeland (Haworth 1994; Whitwell 1990). Neoliberale Theoretiker und Institutionen sind darauf bedacht, daß das Experiment als Erfolg angesehen und die SAP-»Behandlung « auch für andere westliche Länder angewandt wird. Seitdem verkünden die Wächter der Weltwirtschaft das neuseeländische SAP als den einzigen Weg nach vorn für die stagnierenden Marktwirtschaften der fortgeschrittenen Länder. Der Economist beispielsweise besteht darauf, daß das SAP ein Programm von »wegweisenden Reformen« sei, das auch andere westliche Länder mit Rezepten für eine wirtschaftliche Wiederbelebung versorge (zit. $\mathbf{n}$. Kelsey 1995: 8). Das Weltwirtschaftsforum versichert, daß die neuseeländischen Reformen »in der Zukunft weltweit studiert und nachgeahmt werden« (The Australian Financial Review 30.5.96).

1993 bemerkte der Vorsitzende des Runden Tisches der neuseeländischen Wirtschaft, eines mächtigen Zusammenschlusses von Unternehmervertretern, daß Länder mit großen staatlichen Sektoren, wie Kanada, Schweden und Deutschland, »von der Realität eingeholt worden sind « und bald von einer wirtschaftlichen Krise überholt würden (Otago Daily Times 16.10.93). Ihre einzige Retrung, sagte er, läge in der sofortigen Übernahme des SAP. Größenwahnsinnige Vorstellungen hatten Hochkonjunktur, etwa daß die wirtschaftliche »Erholung « die politische (und intellektuelle) Atmosphäre im Land bereichern würde. 1993 stellte die neuseeländische Regierung die Asia 2000-Strategie zusammen, ein Bericht, der für Neuseeland eine großartige Zukunft innerhalb des asiatischen Einflußbereichs versprach.

Das Neuseeland-Wunder ist eine explizit normative Vision. Es ist nicht die prophetische Vision davon, wohin die Entwicklung gehen wird, sondern die Offenbarung von oben, wie die Dinge sein sollten. Das »Wunder « ist eine Sammlung von Tafeln, die vom Berg der ökonomischen Weisheit heruntergekommen ist. (Obwohl die Linke stöhnen wird, daß dieselben Wünsche in der Vergangenheit schon von vielen Bergen herabgestiegen sind.) Für seine Meister ist Neuseelands SAP ein Rezept für eine starke ökonomische Medizin, das befolgt werden muß, wenn kapitalistische Länder Wachstum und einen gesunden Haushalt zurückgewinnen wollen.

So wird der neuseeländische Weg von seinen Befürwortern nicht als unvermeidlich dargestellt; vielmehr erklären sie das SAP als einen Kurs, der nur durch eine dramatische politische Entscheidung von den einzelnen $\mathrm{Na}$ tionalstaaten eingeschlagen werden kann. Allerdings muß man sagen, daß 
die neoliberalen Verfechter des Modells das SAP als die höchste Verkörperung der Vernunft begreifen. So ermutigt der quasi-religiöse Glaube der Neoliberalen an die Zweckrationalität als einen allgemein menschlichen Charakterzug die Befürworter des Modells, Neuseelands SAP mit der Zukunft des Kapitalismus zu identifizieren. Auf der anderen Seite nährt der fast universelle politische Pessimismus der Linken ein ähnliches, aber melancholisches Gefühl von Schicksal. Kritiker befürchten, daß Neuseelands jüngste Geschichte in der Tat die zunehmend verarmte Zukunft entwickelter kapitalistischer Gesellschaften vorwegnimmt. So versuchen verschiedene neuseeländische Gewerkschaftsaktivisten, Akademiker und Mitarbeiter nichtstaatlicher Organisationen (NGOs), die Vergötterung des SAP durch die weltweiten Medien zu kontern, indem sie erklären, daß das »Wunder« eine Vision des Verhängnisses sei, nicht eine des Wohlstands. Die Kritiker fürchten, daß diesem Modell vom Rest der kapitalistischen Welt nachgeeifert wird (die Weltbank hat inzwischen vielen Entwicklungsländern ein ähnliches »Anpassungsprogramm« auferlegt).

Jane Kelsey beispielsweise, Juraprofessorin in Auckland und Kritikerin des SAP, hat die Australier in breit publizierten Vorlesungen vor der Annahme des Reformmodells gewarnt (vgl. The Canberra Times 26.2.96). Im Februar 1995 veröffentlichte der Verband der Nichtstaatlichen Organisationen Neuseelands (ANGO) weltweit einen Bericht, der die begeisterte Darstellung des SAP konterte, die die Regierung dem UN-Gipfel für Soziale Entwicklung in Kopenhagen vorgelegt hatte. Der ANGO-Bericht wollte der Welt »die Geschichte von 10 Jahren sozialer Verwerfungen erzählen, die der offizielle Report Neuseelands verschweigt« (Kelsey/O'Brien 1995: i). Über mehrere Jahre haben führende neuseeländische Gewerkschafter Arbeiterorganisationen in anderen Ländern vor den schlimmen Folgen des SAP für die Arbeiter gewarnt.

Aber es gibt genügend Gründe, daran zu zweifeln, daß sich die Hoffnungen der Befürworter und die Befürchtungen der Kritiker bewahrheiten werden. Man muß im Gegenteil davon ausgehen, daß Neuseeland jeden Glauben, es liefere eine Vorschau auf die globale Zukunft, enttäuschen wird. Denn es wird sich bald zeigen, daß das Neuseeland der neoliberalen Revolution eine instabile und sich schnell fragmentierende Gesellschaft ist, die das überzeugende globale Modell, das von der Rechten so ersehnt und von der Linken so gefürchtet wird, gar nicht liefern kann.

\section{Die Ursprünge der $\gg$ Krise $\ll$}

Welches war nun die ökonomische und administrative »Krise «, die das SAP lösen sollte? Zunächst muß man mit dem Begriff »Krise« etwas vorsichtig sein, wenn man die Zeit des Übergangs in der neuseeländischen 
Gesellschaft beschreiben will, die in den frühen achziger Jahren begann.

Es gibt kaum einen Zweifel daran, daß der korporative Wohlfahrtsstaat, der sich in Neuseeland nach dem 2. Weltkrieg herausgebildet hatte durch die weltweiten politisch-ökonomischen Veränderungen in den 70er Jahren überholt war und in den frühen 80ern einer grundlegenden Überholung bedurfte. Aber es ist wichtig zu sehen, daß die These von der »Krise« nichts anderes als ein rhetorischer Kunstgriff war, der es den Neoliberalen erlaubte, die Veränderung zu nutzen und umzulenken, indem sie das Gefühl einer nationalen ökonomischen Gefahr kultivierten und sich selbst als die einzige politische Kraft etablierten, die zu Reformen in der Lage sei und daher Staat und Gesellschaft »retten« könne. In den frühen 80ern förderten die Neoliberalen im Verein mit den gefälligen nationalen Medien unaufhörlich ein Gefühl von »nationaler Krise« und heizten so ein vorhandenes Unbehagen über den Zustand der neuseeländischen Wirtschaft und der öffentlichen Finanzen an. Durch geschickten Gebrauch der »Politik der Krise« waren die Neoliberalen in der Lage, jeden Vorschlag zurückzuweisen, der darauf hinauslief, daß Neuseelands Schwierigkeiten eine ReRegulierung erfordern könnten. Stattdessen war diese neue Rechte in der Lage, die De-Regulierung als den einzig gangbaren Weg der Reform darzustellen.

Die Hauptschwierigkeiten Neuseelands begannen in den 70er Jahren, als eine Serie von verheerenden Schlägen, die durch globale Veränderungen verursacht waren, sehr schnell die Grundlage für den wirtschaftlichen Wohlstand, der seit dem 2. Weltkrieg geherrscht hatte, zerstörten. Das Ende des Langen Aufschwungs im Nachkriegskapitalismus beeinträchtigte Neuseeland stärker als die meisten anderen westlichen Volkswirtschaften. Neuseeland war in der Nachkriegszeit ein sehr kleiner und peripherer Teilnehmer des westlichen Wirtschaftssystems, und es hing stark vom Handel mit einigen wenigen Schlüsselnationen $a b$, insbesondere Großbritannien und den Vereinigten Staaten. Großbritanniens Beitritt zur Europäischen Gemeinschaft in den frühen 70er Jahren führte zu einem Einbruch in der Nachfrage nach Neuseelands Agrarprodukten. Der Beginn der Weltwirtschaftskrise Mitte der 70er Jahre, im Verein mit der Rückbesinnung des britischen Handels auf Europa, destabilisierte sehr schnell die »entwickelte Abhängigkeit«, die Neuseeland mit Großbritannien kultiviert hatte. Als sich die Handelsprobleme häuften, schnellten Arbeitslosigkeit und Inflation in die Höhe, und der Druck auf die Staatsfinanzen wuchs dramatisch an. Im Klima einer »stagflationären« Rezession begann der Aufstieg der neoliberalen Ideologie; zunächst innerhalb der großen Parteien und der staatlichen Bürokratien, indem verwegene und fertig gepackte »Lösungen« - wie etwa der Monetarismus - für die Doppelkrise angeboten wurden, die Staat und Wirtschaft ergriffen hatte. 
Bis in die siebziger Jahre hatte Neuseeland den umfassendsten Wohlfahrtsstaat der englischsprechenden Welt entwickelt. Im Gefolge der Großen Depression entstanden, wurde Neuseelands Wohlfahrtsstaat auf einer informellen politischen Übereinkunft zwischen Arbeit und Kapital gegründet, die auf stabiles ökonomisches Wachstum und sozialer Sicherheit für alle Bürger zielte. Das regulative System, durch welches dieses Ziel verwirklicht werden sollte, umfaßte starke wirtschaftliche Eingriffe im Inland (Einkommens- und Finanzkontrollen sowie Subventionen im Produktionsbereich) und protektionistische Maßnahmen nach außen. Die »soziale Infrastruktur « beinhaltete umfassende, staatlich finanzierte Systeme der Einkommenssubventionierung, Erziehung, Gesundheit und Wohnungswesen. Zusätzlich wurde das Ziel sozialer Sicherheit durch ein System zentraler Lohnfestsetzung und Schlichtung ergänzt.

So untergrub die plötzliche Unfähigkeit der neuseeländischen Wirtschaft, von der Mitte der siebziger Jahre an ein Einkommen im Außenhandel zu erwirtschaften, die Fähigkeit des Staates, für seine umfassenden sozialen und regulativen Verpflichtungen aufzukommen. Außerdem hatte das »interventionistisch-protektionistische« System eine weitgehend agrarische Wirtschaft hervorgebracht und damit einen Agrarsektor, der von Subventionen abhing und gegen Veränderungen resistent war. Es hatte sich ein kleiner unterentwickelter Industriesektor herausgebildet, der aber im großen und ganzen ein Protektionsprodukt war und lediglich der Binnennachfrage diente. In dem Maße, wie das Außenhandelseinkommen fiel, wuchsen die Staatsschulden dramatisch an - 1984 hatte die jährliche Neuverschuldung 9\% des Bruttosozialprodukts erreicht, und internationale KreditRating-Agenturen warnten davor, daß die Staatsschulden ein Niveau erreichen könnten, das eine Rückzahlung unmöglich würde.

Bis zu den späten Siebzigern hatten die beiden großen politischen Parteien, "Labour « auf der Linken und »National《 auf der Rechten, das protektionistisch-interventionistische Programm als nationale ökonomische Lehrmeinung unterstützt. Tatsächlich versuchte während der frühen achtziger Jahre die amtierende National Party Regierung die Geschwindigkeit des wirtschaftlichen Abstiegs durch »heroische« Interventionsmaßnahmen zu drosseln, einschließlich eines Lohn- und Preisstops und rigider Währungskontrollen. Beim Zurückgreifen auf konventionelle - tatsächlich anachronistische - Formen der Intervention untergrub die National-Regierung die politische und öffentliche Unterstützung für Regulierungsmaßnahmen insgesamt. So faßte in den jüngeren Reihen der oppositionellen Labour Party die Ideologie des Neoliberalismus zuerst Fuß. Einige junge Labourpolitiker waren regelrecht hypnotisiert von den attraktiven und einfachen monetaristischen Konzepten, die von einer neuen, aufsteigenden Generation von neoliberalen Bürokraten und wichtigen Geschäftsleuten vertreten wurden. 
Als die in wachsendem Maße unpopuläre National-Regierung von einem politischen Mißgeschick zum nächsten taumelte, begann eine kleine, aber einflußreiche Gruppe von oppositionellen Politikern zusammen mit neoliberalen Bürokraten in Schlüsselpositionen, den bevorstehenden Machtantritt der Labour-Party zu planen. Nicht einmal die Hierarchie der Labour war sich über das von dieser Gruppe geplante, radikale Reformprogramm in Richtung freie Marktwirtschaft im klaren.

1984 wurde dann eine erklärtermaßen »reformorientierte« Labour-Regierung gewählt. Die öffentliche Meinung war für einen Wechsel, obwohl diese Stimmung kaum homogen war und tatsächlich eine überdeterminierte, manchmal auch widersprüchliche Ansammlung von kulturellen und politisch-ökonomischen Hoffnungen verkörperte. So reflektierte die »Reform «-Stimmung, die die Bildung der neuen Regierung begleitete, tief verwurzelte, wenn auch ambivalente Hoffnungen auf einen kulturellen Wandel - insbesondere auf die Befreiung der neuseeländischen Gesellschaft vom herrschenden Konservativismus, der lange von der staatlichen Politik unterstützt worden war. Viele politische Energien wurden während dieses Prozesses freigesetzt; neuen gesellschaftlichen Bewegungen, insbesondere den Frauen-, den Umweltschutz- und den Maori-Bewegungen (Neuseelands ursprünglicher Bevölkerung) wurden eine erweiterte politische, kulturelle und gesetzliche Anerkennung durch die neue LabourRegierung garantiert. Diese fortschrittlichen kulturellen Reformen dominierten zeitweise die öffentliche Diskussion. Zur selben Zeit gewann die Gruppe der jungen neoliberalen Labourpolitiker wachsenden politischen Einfluß und eroberte Schlüsselpositionen in den Ministerien einschließlich des Finanzministeriums. Da große Teile der neuen Regierung und der Parteiführung durch den »Rausch der kulturellen Reformen « abgelenkt waren, konnten die neoliberalen Minister und Bürokraten die radikale Liberalisierungsstrategie - das SAP - schnell und mit nur wenig politischen Auseinandersetzungen einführen. Als die öffentliche Aufmerksamkeit sich diesen Reformen und ihren Konsequenzen in den späten achziger Jahren wieder zuwandte, verlor Labour die Massenunterstützung und wurde in der Wahl von 1990 deutlich von der National Party besiegt.

So dauerte die erste Phase des SAP von der Wahl der Labour-Party 1984 bis zu ihrer Niederlage 1990 (was zwei Amtsperioden entspricht). Während dieser Zeit beinhaltete das SAP vor allem Deregulierungen (besonders im Finanz- und Handelsbereich) und die stärker unternehmerische Ausrichtung (in einigen Fällen auch Privatisierung) von Staatsbesitz und staatlichen Funktionen. Obwohl neoliberale Minister eine Reduzierung der staatlichen Verpflichtungen im Gesundheitswesen, der Wohlfahrt und im Wohnungswesen anstrebten, wurden sie von einer zunehmend argwöhnischen Parteihierarchie und -organisation an entsprechenden Veränderun- 
gen gehindert - tatsächlich wurden einige neoliberale Minister in den späten Achzigern aus ihren Ämtern entfernt, um so eine Verurteilung ihrer Politik durch die Wähler zu verhindern, was aber am desaströsen Ausgang der Wahlen von 1990 nichts mehr ändern konnte. ${ }^{1}$

Die zweite Phase des SAP begann 1990 mit der von der National Party gestellten Regierung. National bestritt die Wahl mit angeblicher Opposition zum Neoliberalismus von Labour und versprach sogar die Aufhebung einiger Reformen. Nach der Wahl allerdings verriet die National-Regierung diese Versprechen vollständig und erweiterte das SAP, indem sie gewaltige Einschnitte in die soziale Infrastruktur machte und den Arbeitsmarkt deregulierte.

\section{Das »strukturelle Anpassungsprogramm《}

James (1992) und Kelsey (1995) liefern gründliche Rückblicke auf das SAP. Beide beschreiben es als umfassende Restrukturierung von Neuseelands öffentlichem Sektor und der Wirtschaft, die mit der Wahl der Labour-Regierung 1984 begann. Castle und Haworth (1993) zeigen, daß das SAP von einer mächtigen Gruppe von Bürokraten (besonders im Finanzministerium), Politikern und Geschäftsleuten sorgfältig eingefädelt wurde, die sich in den frühen Achzigern aufmachten, um Neuseelands schuldengeplagten Wohlfahrtsstaat entsprechend neoliberalen Grundsätzen umzubauen. Andere Kommentatoren haben diese neoliberale Front mit den Lobbys der Neuen Rechten in den USA und Großbritannien verglichen (vgl. Forer 1995). Mc Loughlin (1995) beschreibt Neuseelands Neoliberalismus als »cargo cult«, angeführt von lokalen Eliten, die der Chicago School eine außerordentliche Ergebenheit bewiesen haben.

Wie schon erwähnt, waren die Hauptbestandteile des SAP eine radikale Einschränkung der öffentlichen Ausgaben und der politischen Regulierung, die Privatisierung von Staatsvermögen und staatlichen Funktionen, die Liberalisierung des Handels und die Deregulierung des Arbeitsmarktes. Neben der Deregulierung des industriellen und finanziellen Sektors der Wirtschaft wurden durch die Reformen auch zentrale und lokale öffentliche Aufgaben wie z.B. Umweltplanung, Erziehung und soziale Dienstleistungen umstrukturiert. Die Dominanz der Ideologie des Freien Marktes, das Streben nach Effizienz bei der Nutzung von Ressourcen und der Einfluß von Beamten des Finanzministeriums sind Kennzeichen des SAP gewesen (Holland/Boston 1990).

1 Diese »Säuberung« wurde sowohl durch Entlassungen als auch durch »ermutigte« Rücktritte bewirkt. Für viele desillusionierte Labourpolitiker und -unterstützer reichten diese Veränderungen nicht aus, da der neoliberale Einfluß auf die Partei bestehen blieb. Konsequenterweise brach eine bedeutende Gruppe der Linken zu dieser Zeit mit der Partei und bildete die New Labour Party, die heute ein Hauptbestandteil der Alliance-Party, einer Regenbogenkoalition verschiedener linker und grüner Gruppen ist. 
Die Ergebnisse des SAP sind allerdings recht unterschiedlich, eine Tatsache, die den Eliten aus Staat und Wirtschaft nicht sehr gelegen ist, da sie das »Experiment « ja als großartigen Erfolg verkaufen. Ein selektiver Blick zurück auf die positiven ökonomischen Indikatoren der letzten Jahre ermöglicht jedoch eine begeisterte Lektüre bei Neoliberalen und Regierungen, die an ihren trägen Volkswirtschaften verzweifelt sind. Die öffentliche Verschuldung konnte reduziert werden - von $51 \%$ des Bruttosozialprodukts 1992 auf 38\% im Jahr 1995, mit der Aussicht auf eine weitere Senkung - während die Regierung seit 1994 nach einer Folge von Defiziten in früheren Jahren beeindruckende Haushaltsüberschüsse erzielen konnte (Kelsey 1995). In den zwei folgenden Jahren nach 1993 gab es ein starkes Wachstum des Bruttosozialprodukts (bis zu 6,7\% im Juni 1994), während die Inflation nach OECD-Standards seit den früheren Neunzigern niedrig geblieben ist. »Rigiditäten « auf dem Arbeitsmarkt (d.h. Gewerkschaften, zentrale Schlichtung und staatliche Anerkennung von Löhnen und Arbeitsbedingungen) waren aufgehoben oder reduziert worden. 1996 war der gewerkschaftliche Organisationsgrad auf etwa 23\% der Beschäftigten gesunken. Die Zahl der Arbeitsplätze erhöhte sich ab 1993 und im Juni 1995 betrug die offizielle Arbeitslosenquote $»$ nur $\ll 6,3 \%$.

Allerdings muß berücksichtigt werden, worauf Easton und Gerritsen (1996) hinweisen, daß die »Erholung « von Wirtschaft und der Staatsfinanzen in den letzten Jahren das Land lediglich für die sieben Jahre wirtschaftlicher Stagnation und sozialen Rückschritts entschädigen, die das SAP den Neuseeländern auferlegte. Viele der wichtigen ökonomischen Indikatoren, wie die öffentliche Verschuldung, erreichen gerade das Niveau von vor 1984 (Easton 1994). Darüberhinaus gibt es zunehmend Hinweise, daß die Erholung bereits ins Stocken kommt, und daß sich eine neue Rezession abzeichnet.

\section{»Das Wachstums/Rezessions-Karussell«: Die ökonomischen Kosten}

In der ersten Phase des SAP (1984-1990), während zweier Labourregierungen, war das wirtschaftliche Wachstum beinahe zum Stillstand gekommen (Wilkes/O'Brien 1994; Roper 1993). In den ersten Jahren nach ihrer Wahl 1990 etablierte die National-Regierung mittels dramatischer Einschnitte bei den öffentlichen Ausgaben ein rezessives makroökonomisches Regime (Dalziel 1992). 1991 schrumpfte die neuseeländische Wirtschaft um 2\%. Obwohl die Wirtschaft seit 1992 wieder wächst, hat das Land immer noch nicht sein Wachstumsniveau von 1985-1986 erreicht. Das SAP reduzierte in Wirklichkeit das Wachstum für längere Zeit: von 1985-1992 expandierten die OECD-Volkswirtschaften insgesamt um 20\%, während die Wirtschaft Neuseelands um 1\% schrumpfte (Easton/Garritson 1996; 
OECD 1993). 1994 charakterisierte der Ökonom Easton das SAP als ein Jahrzehnt verlorenen Wachstums, das durch die Erholung nach 1993 nicht wiedergutgemacht werden konnte (Easton 1994).

Jetzt scheint Neuseelands »turnaround economy« ihren Anhängern auf die Füße zu fallen: Es sieht so aus, als würde die gefeierte »Erholung « von 1994 bereits wieder in sich zusammenfallen, bevor sie richtig angefangen hat. Verschiedene wichtige Makroindikatoren verschlechtern sich seit Anfang 1995 und Wirtschaftsexperten befürchten übereinstimmend eine gröBere Rezession (The New Zealander 8.5.96). Das Bruttosozialprodukt stürzte ab: Im Dezember 1995 war die jährliche Wachstumsrate auf gerade etwas mehr als die Hälfte (3,5\%) des Niveaus von 1994 (6,3\%) zurückgegangen. Anfang 1996 sagten sowohl Ökonomen der Regierung als auch private Wirtschaftsforscher eine weitere Senkung der Wachstumsrate voraus, während das Finanzministerium noch eine Wachstumsrate von 1,5\% zur Jahresmitte prophezeite (The Australian 9.4.96). Der Rückgang im Wachstum hat die Gewinne, das Vertrauen der Unternehmer und die Schaffung neuer Arbeitsplätze spürbar beeinträchtigt. Die düsteren Vorhersagen wurden im März 1997 bestätigt, als die Wirtschaft tatsächlich zu schrumpfen anfing und eine vierteljährliche Wachstumsrate von $-0,5 \%$ festgestellt wurde (The Press 1.7.97). Dementsprechend erwartet die Regierung jetzt einen Anstieg der Arbeitslosenquote auf beinahe 7\% für Ende 1997, was eventuell noch zu optimistisch ist.

Ein Hauptbestandteil des SAP war das Zentralbank-Gesetz von 1989, welches die Zentralbank auf »Preisstabilität« als ihr alleiniges Ziel verpflichtete. Von 1990 an definiert die Regierung »Preisstabilität« als eine jährliche Inflationsrate zwischen 0 und 2\%. 1993 schien es, daß die Bank ihre Aufgabe mit bei einer Inflationsrate von 1,5\% erfüllt hatte (Kelsey 1995). Doch die »Erholung « nach 1992 brachte zunehmenden Druck auf die Preise: 1995 brach eine milde Panik an den Finanzmärkten aus, nachdem die Veröffentlichung der vierteljährlichen Zahlen im Juni eine jährliche Inflationsrate von $4,6 \%$ anzeigte. Fast ein Jahr später mußte der Direktor der Zentralbank zugeben, daß die Inflation immer noch über dem Zielkorridor liegt und auch für den Rest des Jahres dort bleiben würde (The Independent 26.4.96).

Die systemischen Widersprüche des SAP-Modells wurden deutlich als sich die Folgen der dauernden Kürzungen von Investitionen in die Infrastruktur, die Berufsausbildung und die industrielle Forschung zeigten und ihren Tribut von der wirtschaftlichen Aktivität forderten. Eine andere ernsthafte Wachstumsbedrohung tauchte 1995-1996 auf, als die Zentralbank die Geldzügel bei einem (mißglückten) Versuch, die Inflation zu bewältigen, straffte. Hohe Zinsen und eine aufgewertete Währung beeinträchtigten Wachstum und Wettbewerbsfähigkeit, ohne jedoch die Inflation in den 
Zielkorridor zurückzubringen. Der Exportsektor litt im Jahr 1995 schwer: Bis zum Februar 1996 verzeichnete das Land ein Handelsbilanzdefizit von 860 Millionen NZ-Dollar, eine krasse Umkehr des 507 MillionenÜberschusses des vorhergehenden Jahres. Gleichzeitig verschlechterte sich die Zahlungsbilanz erheblich, so daß es 1995 zu einem Defizit von 3,8 Milliarden NZ-Dollar kam. Die Führung der nationalen Agrarlobby argwöhnte sogar, daß selbst dieses gigantische Defizit noch eine Untertreibung war und beschuldigte die Regierung, sie würde die Statistiken frisieren (Otago Daily Times 16.4.96). 1996 verschlechterte sich die Lage des Exportsektors weiter, und das Finanzministerium prognostizierte, daß sich das laufende Zahlungsbilanzdefizit 1998 auf alarmierende 5,2\% des Bruttosozialprodukts erhöhen würde. Diese Befürchtungen schienen sich im ersten Quartal 1997 zu bestätigen, als das Defizit auf 4,8\% des Bruttosozialprodukts gestiegen war (The Press 12.7.97).

Die sich verschlechternde außenwirtschaftliche Situation wurde z.T. auf Privatisierungen und die unkontrollierten ausländischen Investitionen zurückgeführt, die zentrale Bestandteile des SAP waren. 1996 waren etwa $54 \%$ der neuseeländischen Aktien in ausländischem Besitz, 1989 waren es nur 19\% (The Australian Financial Review 5.1.96), und die linke Alliance Party behauptete, daß die »höheren Gewinne der ausländischen Gesellschaften ... zum Defizit beigetragen hätten « (Alliance 1996: 2). Andererseits wurde auch die Zentralbank als weitgehend Verantwortliche ausgemacht, ihr zu strenger antiinflationärer Kurs habe die schwache Erholung nach 1992 untergraben. So bemerkte Peter Harris, ein Wirtschaftsexperte der Gewerkschaften verärgert: »Man sagte uns, daß der Lohn für die Preisstabilität ökonomisches Wachstum sei. Aber jetzt muß das Wachstum gebremst werden, weil es die Preisstabilität gefährdet« (zit. n. Kelsey 1995:170).

Kelsey behauptet, daß beinahe zwölf Jahre nach seinem dramatischen Beginn das SAP Neuseeland in einem »Wachstums/Rezessions «-Karussell gefangenhält (Kelsey 1995: 169). Die »schlanke« Wirtschaft, die die SAPArchitekten absichtlich konstruiert hatten, erweist sich als schlanker und verletzlicher als beabsichtigt. Die Deregulierung hat Neuseeland den Launen der Weltwirtschaft in ganz besonderer Weise ausgeliefert: Der Sektor von ganz besonderer Bedeutung, die Landwirtschaft, wurde durch eine Kombination von frei-floatendem (und überbewertete) NZ-Dollar und fallenden Preisen getroffen. Genauso bedenklich war der Rückgang der wichtigsten Wachstumsbranche der Wirtschaft, des internationalen Tourismus. Er sank Anfang 1996, als andere Länder in den Wettbewerb um den »sauberen, grünen« Reisemarkt traten. 1997 hatte sich der Verlust in den Tourismuseinnahmen in einer sich ohnehin schon verschlechternden Bilanz negativ bemerkbar gemacht (The Press 12.7.97). 
Selbst die langjährigen Freunde scheinen mittlerweile der Unfähigkeit des Modells überdrüssig, die lange versprochenen Erwartungen zu erfüllen. In einem außergewöhnlichen Gefühlsausbruch verglich der Präsident der neoliberalen Farmervereinigung im April 1996 Neuseelands Wirtschaft mit der von Mexiko kurz vor dem verheerenden wirtschaftlichen Zusammenbruch (Otago Daily Times 16.4.96). Eine weitere Bestätigung für bevorstehende Schwierigkeiten kam von einer gänzlich unerwarteten Seite, als die OECD - seit langem eine Befürworterin des SAP - Anfang 1996 vorhersagte, daß Neuseeland 1997 das schlimmste Zahlungsbilanzdefizit der westlichen Welt haben würde (The Age 10.2.96). Im Juni 1996 hatte Neuseelands Nettoauslandsverschuldung (privat und öffentlich) $65 \%$ des Bruttosozialprodukts erreicht, höher als in irgendeinem entwickelten Land (The Press 31.5.96). Ein Jahr später dauerte die Verschlechterung der AuBenbilanz mit nur geringer Aussicht auf Besserung an - im ersten Quartal 1997 hatte das Land ein Defizit von 723 Millionen NZ-Dollar.

\section{»Gespenster beim Bankett«: Die gesellschaftlichen Kosten}

Das ganze Jahr 1990 hindurch versuchten die erst kürzlich kommerzialisierten Medien des Landes die Unterstützung für einen neuen nationalen, verbindenden Mythos zu beschwören - das Ideal vom "Team New Zealand . Das geschah durch einen Sportrummel, der durch eine zunehmend aggressive Prahlerei charakterisiert war. Aber trotz einiger bemerkenswerter internationaler Sporterfolge verfehlte die Kampagne ihre Wirkung: Das »Team New Zealand « ist zutiefst uneinig und durch die Veränderungen verstört (James 1992:5). Viele Menschen erinnern sich an eine glücklichere Zeit in der Vergangenheit: als alles anders war; als es schien, daß Neuseeland das Gelobte Land (»Godzone «) sei.

Der »Godzone «-Mythos konserviert das Bild vom verlorenen Neuseeland: einer friedlichen egalitären Gesellschaft, wo die üblichen Krankheiten entwickelter Nationen - Verbrechen, Armut, Umweltverschmutzung und Rassenkonflikte - so weit entfernt waren wie die übrige Welt selbst (James 1992; Mc Loughlin 1992). Etwas von dem ländlichen Idyll taucht in dem vernebelten Bild von Neuseeland auf, einem Arkadien des natürlichen Überflusses, in dem die Leute ihr unkompliziertes und ruhiges Leben lebten. Die Gesellschaft von »Godzone« war intim und harmonisch, geleitet von kulturellen Normen gegenseitiger Unterstützung und Verpflichtung, ein Erbe der zähen britischen Siedler des Landes aus dem 19. Jahrhundert. Die Geschichte von »Godzone« erzählt dann, wie diese Pioniertugenden der gegenseitigen Unterstützung und der Gleichheit während der dreißiger Jahre des 20. Jahrhunderts im ersten Wohlfahrtsstaat der Welt kodifiziert wurden, der schließlich das umfassendste Netz sozialer Unterstützung in 
der englischsprechenden Welt einrichtete. Soziale Sicherheit wurde sowohl durch ein hohes Niveau von Solidarität als auch durch einen fürsorglichen Staat garantiert.

Verschiedentlich wurde schon darauf aufmerksam gemacht, daß die reiche Ausschmückung des »Godzone«-Ideals die lange Geschichte von Gewalt, Landenteignung und gesellschaftlicher Unterentwicklung verdeckt, die von den Maori, der indigenen Bevölkerung des Landes, seit der Kolonialzeit erlitten wurde (vgl. Spoonley u.a. 1991). Andere Autoren (vgl. z.B. Bedggod 1980; Wilkes 1994) haben die liebevoll gehegte Idee von »Godzone « als einer klassenlosen Gesellschaft kritisiert, indem sie sowohl die Existenz eines beträchtlichen Proletariats als auch einer weitgehend verarmten Schicht seit der weißen Besiedlung aufzeigten. Während diese und andere Kritiken des »Godzone«-Mythos sicherlich berechtigt sind, stimmt es trotzdem, daß Neuseeland vor 1984 eine vergleichsweise konsensorientierte, insulare Gesellschaft war - zumindest nach internationalen Standards. Forer (1995:251) charakterisiert das Neuseeland von vor 1984 als »eine bemerkenswert fürsorgliche und persönliche Gesellschaft«. Zwölf Jahre SAP haben das alles verändert.

Die Restrukturierung des öffentlichen Sektors war und ist ein kritischer Bereich des SAP. Seit 1984 hatten dramatische »Reformen « in allen Teilen des öffentlichen Sektors das Ziel, die angebliche Abhängigkeit des einzelnen Neuseeländers vom Staat zu reduzieren. Grundsätzlich hat die Restrukturierung fiskalische Einschnitte gebracht und den Anspruch auf soziale Unterstützung beschränkt; außerdem führte sie zu einem Wechsel in Richtung auf eine indirekte Besteuerung, Benutzergebühren für öffentliche Dienste und Senkung sozialer Hilfen. Auch wurden viele staatliche Funktionen im Bereich Gesundheit, Erziehung, Wohnungswesen und Wohlfahrt als Unternehmen organisiert und häufig privatisiert (vgl. Kelsey/O'Brien 1995 und Boston/Dalziel 1992 für einen umfassenden Überblick). Die radikale Beschneidung des öffentlichen Sektors erreichte ihren Höhepunkt mit dem Budget der National-Regierung von 1991, in dem das Niveau für die Hilfe an Arbeitslose, Kranke und Alleinerziehende drastisch eingeschränkt wurde. Die Einschnitte bewegten sich zwischen schmerzhaft und bösartig: Die Zahlungen für junge Arbeitslose beispielsweise wurden real um 25\% gekürzt. Die Einsparungen von 1991 konsolidierten auf dramatische Art und Weise die Einschnitte bei der Wohlfahrt, die schon von den früheren Labour-Regierungen nach 1984 eingeleitet wurden. Kelsey kommentiert, daß das Budget von 1991 »die Schichtung der neuseeländischen Gesellschaft formalisierte, die Labour begonnen hatte « (1995: 273).

Das SAP griff das korporative Wohlfahrtsmodell auch an einer anderen Stelle durch die Deregulierung der Arbeitsbeziehungen an. Das Beschäftigungsgesetz von 1991 fegte das alte System zentraler Schlichtung und 
Lohnfestsetzung vollständig weg und ersetzte es durch einen liberalisierten, durch Einzelverträge geregelten Arbeitsmarkt, der Autorität und Rolle der Gewerkschaften bei den Verhandlungen drastisch reduzierte. Für die Geschäftswelt hatte dieses Gesetz unerwartete und sehr angenehme Resultate: Die Mitgliederzahl der Gewerkschaften ging nach 1991 schnell zurück, Löhne und Arbeitsbedingungen verschlechterten sich und die Arbeitsunsicherheit wuchs. Der anhaltende Verlust bei den durchschnittlichen Reallöhnen seit 1991 wurde von Lohndiversifizierungen und einer Verstärkung der sozialen Schichtung begleitet (ACROSS-ACTU Study Program 1996).

Die Restrukturierung des staatlichen Sektors und der Arbeitsmärkte hat für viele Menschen den Lebensstandard gesenkt (St John 1994). Tatsächlich bezeichnen O'Brien und Wilkes (1993) das SAP als eine »Verarmungspolitik«. Auch eine von der Regierung in Auftrag gegebene Untersuchung stellte fest, daß einer von fünf Neuseeländern in Armut lebt ${ }^{2}$ (Otago Daily Times 12.4.96). Dieselbe Studie schätzt, daß beinahe ein Drittel der neuseeländischen Kinder unterhalb der Armutsgrenze lebt. Ein weiteres Barometer zur Feststellung der Armut, die Nachfrage nach karikativer Unterstützung, hat einen schockierenden Anstieg des sozialen Elends aufgezeigt. $1994 \mathrm{gab}$ es in Auckland 130 Essensausgabestationen (ein relativ neues Phänomen in Neuseeland), die pro Monat etwa 5000 Pakete verteilten zehnmal so viel wie 1990 (Otago Daily Times 15.9.94). In Dunedin auf der Südinsel berichtete eine größere Wohlfahrtsagentur 1995 von einem Anstieg von $1000 \%$ bei der Anzahl der Nahrungspakete, die in den vorangehenden sechs Jahren verteilt wurden (Otago Daily Times 11.9.95).

Im heutigen Neuseeland verbreitet sich auch zunehmend das »ausländische« Übel der »Arbeitsarmut«. Eine jüngste Untersuchung der Heilsarmee über die Benutzer der Essensausgabestationen fand heraus, daß beinahe $11 \%$ Arbeit hatten - in einigen Gegenden sogar 20\%. Der Arbeitsminister tat die Untersuchungsergebnisse mit einem Schulterzucken $a b$ und bemerkte lediglich, daß »einige Jobs eben nicht viel wert sind « (Otago Daily Times 24.1.96). Einige Führungskräfte aus dem privaten Sektor waren da hilfreicher. Als Reaktion auf die Berichte von Lehrern über zunehmenden Hunger unter jüngeren Schülern drängte der stellvertretende Vorsitzende des Runden Tisches der Wirtschaft die Schulen, für Eltern-Klassen die Zubereitung von Sandwiches einzurichten, um »die Versorgung der Schüler zu verbessern « (Otago Daily Times 26.3.94).

Anfang 1995 veröffentlichte die britische Joseph Rowntree Foundation eine verläßliche Studie über Veränderungen der Einkommensunterschiede in

2 Als Armutsgrenze gelten 60\% des »Median-Einkommens« (d.h. desjenigen Haushaltseinkommens, in dessen Umgebung die meisten Haushaltseinkommen liegen). 
18 entwickelten Ländern im Verlauf der achziger Jahre. Der Studie gemäß verzeichnete Neuseeland den größten Zuwachs bei der Ungleichheit der Einkommen und überholte sogar die USA und Großbritannien (Joseph Rowntree Foundation 1995). Auch Rankin (1995) bringt Daten, die den dramatischen Zuwachs bei den Einkommensunterschieden in Neuseeland seit 1984 zeigen. Sogar der Economist kam zu dem Schluß, daß der Unterschied zwischen arm und reich einer der höchsten der Welt geworden war. Für die Befürworter des SAP eher beunruhigend, meinte der Economist, daß solche Einkommensunterschiede das wirtschaftliche Wachstum erstikken könnten. Aber die Regierung blieb unerschütterlich, um nicht zu sagen ungeniert, während immer deutlicher wurde, daß das SAP Neuseelands Gesellschaft zu zerreißen drohte.

Eine ganze Reihe von Indikatoren signalisiert ein potentiell explosives Unbehagen in der neuseeländischen Gesellschaft. Die SAP-Reformen haben sowohl eine fortwährend wachsende und an den Rand gedrängte Schicht von Armen, Arbeitslosen, Kranken und schlecht Wohnenden, als auch eine blühende Untergrundwirtschaft erzeugt. Die Verurteilungen für schwere Verbrechen stiegen zwischen 1982 und 1991 um erschreckende $50 \%$ an (Kelsey 1995). 1994 kam eine Studie des Britischen Innenministeriums zu dem Schluß, die Wahrscheinlichkeit, daß Neuseeländer Opfer eines Verbrechens würden, sei größer als in irgendeinem anderen industrialisierten Land (Otago Daily Times 22.4.96).

Arbeitslosigkeit und zunehmende Rassenspannungen begünstigten die $\mathrm{Zu}$ nahme gewalttätiger Banden von Jugendlichen und ethnischen Minderheiten, von denen viele weitreichende Verbindungen sowohl zur Untergrundwirtschaft als auch zu kriminellen ausländischen Organisationen, wie den asiatischen Triaden haben. 1996 schätzte man die Anzahl der größeren Banden von Jugendlichen und ethnischen Minderheiten auf 70, mit einer Gesamtmitgliedschaft von etwa 11.000 (Canberra Times 19.5.96). Die polizeilichen Verlautbarungen konnten die Ängste der Bevölkerung kaum beruhigen: Im Mai gab der Polizeipräsident zu, daß die Polizei »nahe daran sei, die Kontrolle über das organisierte Verbrechen zu verlieren« (The Press 31.5.96). Die Behörden unternahmen verzweifelte Versuche, wie etwa die Verhängung von Ausgangssperren, um der Bandenkriminalität zu begegnen, während Politiker und Boulevardpresse über die neue »Bedrohung durch die Jugend « klagten. Inmitten der wachsenden nationalen Hysterie lieferten Mitte 1996 Vertreter der Banden einige rationale Bewertungen des Problems und stellten das SAP als den Grund für die Entfremdung der Jugend und die Rassenspannungen heraus. Der Sprecher einer Bande, des »Mongrel Mob«, wußte genau, was die größte Hilfe für die Rekrutierung seiner Leute war: »Gangs sind ein gesellschaftliches Barometer. Ganz plötzlich war da über eine Zeit von 10 Jahren ein phänome- 
naler Anstieg der Zahl der Gang-Mitglieder, und das zeigt, was in der Gesellschaft abläuft.« Diese Ansicht wurde vom Sprecher der rivalisierenden »Black Power Gang « wiederholt, der provozierend erklärte, »das einzige Verbrechen, das in diesem Land begangen werde, sei das der Regierung « (The Canberra Times 19.5.96).

Die dramatisch gestiegene Selbstmordrate während der SAP-Zeit hat ebenfalls das soziale Elend aufgezeigt: "Zwischen 1974 und 1990 stieg die Selbstmordrate bei Männern um 288\%, mit dem höchsten Zuwachs in den späten achziger Jahren. 1990 hatte Neuseeland die höchste Selbstmordrate bei den 15-24-jährigen Frauen in der gesamten OECD und die dritthöchste bei den jungen Männern « (Kelsey 1995:295).

Die unternehmensorientierte Umwandlung des öffentlichen Gesundheitswesens hat unter anderem die Anhebung der Beiträge für die Grundversorgung und explodierende Rezeptgebühren mit sich gebracht (Fougere 1994). Das Ergebnis war ein erschwerter Zugang zur Basis-Gesundheitsvorsorge für die wachsende Zahl der Armen und das Wiederauftauchen von Armutskrankheiten wie Rachitis, Tuberkulose und Skorbut (Kelsey/ O’Brien 1995).

Die Maori und die Einwanderer der Pazifikinseln haben am meisten unter dem SAP gelitten. Insbesondere die Maori haben viele Jobs bei den Privatisierungen und bei der Restrukturierung der Industrie verloren; auch durch die Kürzungen der öffentlichen Unterstützung wurden sie besonders betroffen. Im Dezember 1993 war ein Drittel der jugendlichen Maori arbeitslos, und fast zwei Fünftel der Maorikinder lebten in Haushalten, die zu den $20 \%$ mit den niedrigsten Einkommen gehörten (Kelsey 1995). Die Maori schnitten auch bei den meisten anderen Negativindikatoren weit schlechter ab als die Nicht-Maori, wie etwa bei der Schwangerschaft von Minderjährigen, der Abhängigkeit von Unterstützung und bei der Selbstmordrate. Im April betrug die Arbeitslosigkeit bei den Immigranten der Pazifikinseln über 21\% (Sunday Star Times 30.4.95).

Das jetzige Neuseeland besteht für Spoonley (1994: 93) aus »zwei Nationen«, die »scharf entlang einer polynesisch/nicht-polynesischen Grenze getrennt« sind. Wie Kelsey (1995) erklärt, sehen viele Maori das SAP in neokolonialen Begriffen, als eine Verstärkung des Prozesses der Enteignung, Verarmung und Entfremdung, der mit der weißen Besiedlung von 1840 angefangen hat. Die extreme Entfremdung der Maori nährt eine neue, höchst militante Politik unter Neuseelands indigenen Völkern. Eine zunehmend einflußreiche und radikale Schicht von jüngeren Maoriführern fordert vollständige Souveränität für ihr Volk. Spannungen zwischen Maori und Nicht-Maori flammten 1995 auf, als es zu einer Serie von dramatischen Land- und Gebäudebesetzungen durch Ureinwohner kam. Wie Kelsey erklärt, »zeigten die Besetzungen eine wirksame Bedrohung der neuen 
Ökonomie«, indem sie die Sicherheit der Eigentumsrechte bedrohten und so die Investoren alarmierten. 1996 dauerte die Verärgerung der Maori über die Folgen der Reformen an, und bei den Kommentatoren wächst ein ungutes Gefühl: Trotter (1995) schrieb über die Möglichkeit eines von Maori geführten Aufstands in Neuseeland, während Kelsey (1995: 322) eine »ausgesprochene Rebellion gegen den Staat« in der nahen Zukunft nicht ausschließen wollte. Der Soziologe Shirley kommt zu folgender pessimistischer Beurteilung des gesellschaftspolitischen Vermächtnisses des SAP:

»Innerhalb unserer gespaltenen Gesellschaft haben wir eine Unterklasse etabliert, die nicht länger ein persönliches Interesse an Demokratie oder demokratischen Institutionen hat, und konsequenterweise sollten wir nicht überrascht sein, wenn solchermaßen vorn gesellschaftlichen Leben Neuseelands Verbannte bereitwillig seine Zerstörung fördern« (zit. n. Kelsey 1995: 296).

\section{$\gg$ Durch Wahlen geschaffene Instabilität«: Die politischen Auswirkungen}

Trotz gesellschaftlicher Fragmentierung und Konflikten wird eine Mehrheit der Neuseeländer durch ein politisches Ziel geeint: die Überwindung des SAP. Tatsächlich ist, seit die Reformen eingeführt wurden, immer eine Mehrheit der Wähler gegen das SAP gewesen, aber dieser Widerstand ist durch die beiden großen Parteien, das Mehrheitswahlrecht und den »Crashkurs « der Reformer unter Kontrolle gehalten worden (James 1992). Der Rekord sowohl von Labour als auch von National beim Brechen der Wahlversprechen, das SAP zu verlangsamen oder zu stoppen, und die überaus schnelle Ausführung des Programms, haben das Vertrauen der Neuseeländer in ihr Wahlsystem und ihre Politiker unterhöhlt. Eine Umfrage Mitte 1992 zeigte, daß immerhin 81\% der Wähler glaubten, die neuseeländische Politik sei korrupt (Kelsey 1995).

Darauf antwortete 1993 die Bevölkerung, indem sie in einem nationalen Referendum das Mehrheitswahlrecht zugunsten eines Verhältniswahlrechts abschaffte. Die erste nationale Wahl unter dem neuen Wahlsystem im Oktober 1996 war vom öffentlichen Zorn über das SAP geprägt. Die amtierende National-Party - Hauptbefürworterin des SAP - wurde von nur 34\% der Wähler unterstützt, während eine Sammlung von Mitte-Links-Parteien, einschließlich einer neuen, zentristischen Labour-Party, etwas über die Hälfte der Stimmen $(52 \%)$ erzielte. ${ }^{3}$

Der Wahlkampf war durch den spektakulären Aufstieg der $\gg$ New Zealand First Party « (NFZ) in den Meinungsumfragen gekennzeichnet, einer neuen populistischen Partei, deren charismatischer Führer die zunehmenden ethnischen Spannungen, die ökonomischen Unsicherheiten und die Abnei-

3 Die Aufschlüsselung der Mitte-Links-Stimmen ergibt: Labour (28,2\%); New Zealand First-Party $(13,4 \%)$; Alliance-Party $(10,1 \%)$. 
gung der Wähler gegenüber den Hauptbestandteilen des SAP, wie Privatisierung und direkte ausländische Investitionen, ansprach. In der Folge erhielt die NFZ nur einen bescheidenen Anteil von 13,4\% der Wählerstimmen. Trotzdem brachte dieses Ergebnis, das drittbeste überhaupt, die NFZ in eine Schlüsselposition bei den Verhandlungen über eine Koalitionsregierung.

Als sich das Wahlergebnis - und die Niederlage von National - abzeichnete, warnte ein ganzer Schwarm von neoliberalen Kadern, angefangen bei den Vertretern der Industrie und den internationalen Finanzjournalisten bis hin zu den Kredit-Rating-Agenturen, davor, daß die durch die Wahlen hervorgerufene Instabilität (d.h. die politische Zurückweisung des SAP) schreckliche Konsequenzen für Neuseelands Wirtschaft bringen würde. Nur ein Jahr vor der Wahl waren die Umfrageergebnisse für die LabourParty so schlecht gewesen, daß viele Kommentatoren ihren bevorstehenden Zusammenbruch und ihr Verschwinden prognostizierten. Doch 1996 bewegte sich die Partei nach links und wurde eine zunehmend wichtige Kritikerin (wenn nicht totale Gegnerin) des SAP. Mit der Wahl wurde Labour mit einer dramatischen Verbesserung ihrer Popularität belohnt, obwohl das Ergebnis für eine Regierungsbildung nicht ausreichte.

In der Folge gab es einen weiteren Fall von politischem Betrug, der so charakteristisch für die SAP-Ära ist. Die NFZ-Party entschied gegen die erklärte Absicht der meisten Mitglieder, die Regierung der National-Party durch Eintritt in die Koalition zu retten. Der Preis für das Überleben von National, den die NFZ gefordert hatte, war die Verlangsamung des SAP und die Rücknahme einiger Maßnahmen. Folgerichtig wurden Ausgabesteigerungen im Gesundheitswesen und bei der Wohlfahrt angekündigt und die Re-Regulierung von bestimmten Politikbereichen versprochen. Doch das erste Budget der Koalition im Juni 1997 enthielt nur sehr wenige zusätzliche Sozialausgaben. Darüberhinaus wies es einen neuen gesellschaftlichen Konservatismus, wenn nicht gar Autoritarismus auf, indem ein »neuer Vertrag« zwischen Staat und Wohlfahrtsemfängern vorgeschlagen wurde. Als Teil dieses Vertrages wurde zum Beispiel angeregt, daß Eltern für Schuleschwänzen oder Nichtimpfenlassen ihrer Kinder mit dem Entzug von Unterstützungsleistungen bestraft würden.

Selbst angesichts dieser antidemokratischen Manipulationen und des Betrugs durch die Mitte-Rechts Parteien hält die Absicht der Öffentlichkeit, das SAP zu beenden, unvermindert an. Im Juli 1997 fiel die NZF in den Umfragen auf $3 \%$, noch unter die Grenze der statistischen Unsicherheit und, noch wichtiger, unter den für die Sitzverteilung beim neuen Verhältniswahlrecht notwendigen Stimmenanteil. Die Unterstützung für die National-Party war auf $33 \%$ gefallen, während die Mitte-Links-Parteien, Labour und Alliance, auf $42 \%$ und $9 \%$ kamen (The Press 12.7.97). Für die 
neoliberalen Anhänger des SAP scheint als einzige Strategie der fortdauernde Wahlbetrug der regierenden Parteien zu bleiben. Wenn man den Übergang zur Diktatur für Neuseeland ausschließ $3 t$, scheint diese Politik aber längerfristig zum Scheitern verurteilt zu sein.

\section{Schluff folgerung: Ein Weltmodell?}

Das Wunder von Neuseeland wird schnell zu einer weiteren Parabel für kapitalistische Restrukturierung. Die Bedeutung des SAP über Neuseeland hinaus schwindet in dem Maße, wie sich dieses Modell an internen Widersprüchen reibt und wie seine geopolitischen Besonderheiten sichtbar werden. Ein Faktor, der gegen die Verallgemeinerung des SAP spricht, ist nämlich gerade seine Anwendung in einer nur teilweise entwickelten Miniatur-Wirtschaft, ${ }^{4}$ die noch dazu am Rande der kapitalistischen Weltwirtschaft liegt.

Die letzten Wahlen haben gezeigt, daß andere Völker - zum Beispiel in Großbritannien, Frankreich, Schweden, Italien und den USA - sehr mißtrauisch gegenüber der neoliberalen Politik sind. Tatsächlich wurden 1997, zum ersten Mal seit Jahren, mehrere neoliberale Hochburgen entweder von außen durch die politische Opposition bedroht, oder von innen durch untypische ideologische Zweifel. In einem Beispiel der letzten Zeit wurde die Durchsetzung der Europäischen Währungsunion mit einer wiedererstandenen Opposition quer über den Kontinent konfrontiert. Selbst die Weltbank, eine globale Bastion des Neoliberalismus, und einstmals Anhängerin des »Wunders von Neuseeland «, hat sich erst kürzlich von allzu marktradikalen Theorie distanziert und stattdessen für einen mstarken und energischen Staat« plädiert (The Guardian 26.6.97).

Langsam scheinen die Propheten aus ihren Träumen zu erwachen. Einer der wichtigsten Apostel des Neoliberalismus der 80er Jahre aus den USA, Stephen S. Roach (der »Guru des downsizing «), machte 1996 eine spektakuläre politische Kehrtwendung und rät nun zu einer quasi-interventionistischen Politik der Regierungen (Roach 1996). Zur selben Zeit hat Francis Fukuyama, der einst das Ende der Geschichte ausgerufen hat, ganz andere Gedanken über die gesellschaftliche Machbarkeit der freien Marktwirtschaft entwickelt (Fukuyama 1995).

Das alles soll nicht heißen, daß das neuseeländische Experiment nicht den politisch-ökonomischen Wandel in der kapitalistisch entwickelten Welt beeinflussen wird. Die »vorläufigen Resultate« haben die Wächter der Weltwirtschaft bereits beeindruckt und ohne Zweifel nationale Restrukturie-

4 Neuseeland hat niemals eine forcierte industrielle Entwicklung durchgemacht - sein industrieller Sektor ist immer unbedeutend gewesen. Der bestimmende Faktor der Nachkriegswirtschaft war die Abhängigkeit von Agrarexporten. 
rungskonzepte andernorts beeinflußt. Aber es gibt nichts so Unpopuläres, nichts historisch so Überflüssiges, wie ein Experiment, das einfach nicht funktioniert. Und das Laboratorium Neuseeland macht immer offensichtlicher, daß das SAP ein Experiment ist, das durch nicht vorhergesehene Ergebnisse und Kehrtwendungen scheitern wird. Whitwell (1990:108) charakterisiert das SAP als einen verfehlten »Appell an die alte Orthodoxie «, und es scheint sicher, daß die Geschichte das neuseeländische Experiment lediglich als eine weitere Fußnote der isolierten Anwendung des alten laisser-faire-Modells betrachten wird.

\section{Übersetzung aus dem Englischen von Günter Barwig}

\section{Literatur}

ACOSS-ACTU Study Program (1996): Report of the Study Program on Structural Adjustment and Social Change: Stage 1- New Zealand, ACTU-ACOSS, Sydney-Melbourne.

Alliance Parliamentary Office (1996): Foreign Investment - Key Facts, unpublished mimeograph, Wellington.

Bedggood, D. (1980): Rich and Poor in New Zealand, Allen \& Unwin, Auckland.

Boston, J. \& Dalziel, P. [eds] (1992): The Decent Society? Essays in Response to National's Social and Economic Policies, Oxford University Press, Auckland.

Castle, R. \& Haworth, N. (1993): The Economic Imperative for Restructuring in Australia and New Zealand' in: Bray, M. \& Haworth, N. [eds], Economic Restructuring and Industrial Relations in Australia and New Zealand: A Comparative Analysis, ACIRRT Monograph no. 8, University of Sydney, S.16-37.

Dalziel, P. (1992): National's Macroeconomic Policy, in: Boston/Dalziel, S.19-38.

Driver, F. (1993): Back to the Future of Geography, Environment and Planning, Anniversary Issue, S.22-5.

Easton, B. (1994): Economy, Listener, 17 December, S.60.

Easton, B. \& Gerritsen, R. (1996): Economic Reform: Parallels and Divergences, in: Castles, F.G., Gerritsen, R. \& Vowles, J. [eds], The Great Experiment: Labour Parties and Public Policy Transformation in Australia and New Zealand, Allen \& Unwin, Sydney, S.22-47.

Forer, P. (1995): Off the Sheep's Back? Two Decades of Change in New Zealand, in: Cliff, A.D. et al. [eds], Diffusing Geography: Essays for Peter Haggett, Blackwell, Oxford, S.241-61.

Fukuyama, F. (1995): Trust: Social Virtues and the Creation of Prosperity, Hamish Hamilton, London.

Haworth, N. (1994): Neo-Liberalism and Economic Internationalisation, in: Sharp, A. [ed], Leap into the Dark: the Changing Role of the State in New Zealand since 1984, Auckland University Press, Auckland, S.19-40.

Holland, M. \& Boston, J. [eds] (1994): The Fourth Labour Government: Politics and Policy in New Zealand, Oxford University Press, Auckland.

James, C. (1992): New Territory: the Transformation of New Zealand 1984-92, Allen \& Unwin, Sydney.

Joseph Rowntree Foundation (1995): Inquiry into Income and Wealth (2 vols.): Joseph Rowntree Foundation, York.

Kelsey, J. (1995): The New Zealand Experiment, Auckland University Press, Auckland.

Kelsey, J. \& O'Brien, M. (1995): Setting the Record Straight: Social Development in Aotearoa/New Zealand, Association of Non Government Organisations of Aotearoa, Palmerston North.

OECD (1993): Economic Outlook, Paris.

OECD (1994): Economic Surveys: New Zealand, Paris. 
Rankin, K. (1995): Whose Getting What? Income Distribution in Godzone, New Zealand Political Review, April/May, S.10-15.

Roach, S. (1996): US: Backlash Over Productivity, in: Global Economic Forum (internet version): 14 May, Morgan Stanley, New York, S.2.

Roper, B. (1993): The End of the Golden Weather: New Zealand's Economic Crisis, in: Roper, B. \& Rudd, C. [eds], State \& Economy in New Zealand, Oxford University Press, Auckland, S.1-25.

St John, S. (1994): The State and Welfare, in: Sharp, A. [ed], Leap into the Dark: the Changing Role of the State in New Zealand since 1984, Auckland University Press, Auckland, S.88-106.

Spoonley, P. (1994): Racism and Ethnicity in: Spoonley, P., Pearson, D \& Shirley, I. [eds], New Zealand Society, Dunmore, Palmerston North, S.81-97.

Spoonley, P., Pearson, D. \& Macpherson, C. [eds] (1991): Nga Take: Ethnic Relations and Racism in Aotearoa/New Zealand, Dunmore, Palmerston North.

Trotter, C. (1995): The Struggle for Sovereignty, in: New Zealand Political Review, April/May, S.16-28.

Whitwell, J. (1990): The Rogernomics Monetarist Experiment in: Holland/Boston, S.101-22.

Wilkes, C. (1993): Class, in: Spoonley/Pearson/Shirley, S.66-80.

Wilkes, C. \& O'Brien, M. (1993): The Tragedy of the Market, Dunmore, Palmerston North.

\section{Prof. Dr. Jürgen Kuczynski}

1904-1997

Wir trauern um unseren Autor. 